\title{
Evaluation of the Effectiveness of Urine Dipstick in the Screening of Urinary Infection in Relation to the Reference Method: The Cytobacteriological Examination of Urine
}

\author{
Bianza Moise Bakadia1*, Bakamona Lina Babidi², Tshilumbayi Falone Mutoba1, \\ Mazono Pierre Mbang ${ }^{1}$, Kabengele Arlette Ndaya', Mutumukole Jeanluc Biampata ${ }^{3}$, \\ Ilunga Eric Kasamba ${ }^{4}$
}

\author{
${ }^{1}$ Department of Medical Biology, Higher Institute of Medical Techniques of Lubumbashi, Lubumbashi, DR Congo \\ ${ }^{2}$ Department of Nursing Sciences, Higher Institute of Medical Techniques of Lubumbashi, Lubumbashi, DR Congo \\ ${ }^{3}$ School of Public Health, University of Lubumbashi, Lubumbashi, DR Congo \\ ${ }^{4}$ University of Lubumbashi, Faculty of Medicine, Lubumbashi, DR Congo \\ Email: ^bakadiafalone@gmail.com
}

How to cite this paper: Bakadia, B.M., Babidi, B.L., Mutoba, T.F., Mbang, M.P., Ndaya, K.A., Biampata, M.J. and Kasamba, I.E. (2018) Evaluation of the Effectiveness of Urine Dipstick in the Screening of Urinary Infection in Relation to the Reference Method: The Cytobacteriological Examination of Urine. Open Access Library Journal, 5: e4790.

https://doi.org/10.4236/oalib.1104790

Received: July 23, 2018

Accepted: August 13, 2018

Published: August 16, 2018

Copyright $\odot 2018$ by authors and Open Access Library Inc.

This work is licensed under the Creative Commons Attribution International License (CC BY 4.0).

http://creativecommons.org/licenses/by/4.0/ (c) (i) Open Access

\begin{abstract}
The cytobacteriological examination of urine is a biological test most commonly asked in the hospital. The results of a large part of urinary sample analyzed are negative, representing a heavy workload for bacteriology laboratories, burden that we would like to limit. In this context the aim of our study was to evaluate a screening test for urinary tract infection by urine dipstick compared to the reference method; the cytobacteriological examination of urine finally promotes the realization of the urine dipstick before any urine culture. We selected 600 patients whom the physician asked the cytobacteriological examination of urine, and on every patient, we proceeded to urine collection and finally realized the urine dipstick before urine culture; two parameters on urine dipstick have helped us to confirm the negativity and positivity of the dipstick, its leukocytes and nitrites. The results of urine dipstick are positive if one or less of the two tests, leukocyte and nitrite is positive; the result is negative if both tests are negative. The urine culture was done on cled agar and bacterial identification was made on cultural characteristics, biochemical (API 2OE gallery of biomerieux). The results of urine culture were collected 72 hours after culture. For the analysis of results, the sensitivity, specificity, predictive values (positive predictive value and negative predictive value), the youden index, the proportion of correctly-classified subjects and the likelihood ratio (positive likelihood ratio and negative likelihood ratio) were calculated. The urine dipstick has the sensitivity of $96 \%$, specificity of
\end{abstract}


$90 \%$, the positive predictive value of $74 \%$, the negative predictive value of $98.8 \%$, youden index of 0.86 , the proportion of subjects correctly classified of $91.6 \%$, a positive likelihood ratio of 9.6 and a negative likelihood ratio of 0.04 . In the light of these results, the urine dipstick is an elimination test, by its very high sensitivity (96\%), its high negative predictive value $(98.8 \%)$ and negative likelihood ratio close to 0 or 0.04 , and if the dipstick does not detect nitrite and leukocyte, the diagnosis of urinary tract infection is highly unlikely $(<2 \%$ probability).

\section{Subject Areas}

Nursing

\section{Keywords}

Urine Dipstick, Urinary Tract Infection, Cytobacteriological Examination

\section{Introduction}

Urinary tract infection is the most common bacterial infection and causes a significant burden on health system resources. In the community, it mainly affects sexually active women but also people of all ages. In the hospital environment, the elderly and the carriers of the urinary catheters are the main affected patients. The concept of a urinary tract infection is broad, ranging from asymptomatic infection to pyelonephritis with sepsis [1] [2] [3] [4]. Urinary tract infections are usually caused by a single microorganism. Escherichia coli is the causative agent in over $80 \%$ of infections and Staphylococcus saprophyticus in 10\% to $15 \%$ of infections. Occasionally, other infectious agents may be involved such as Klebsiella spp., Proteus mirabilis, and Enterococcus faecalis. The etiology of urinary tract infection varies depending on the risk factors and the type of infection (complicated vs. uncomplicated) [5] [6] [7]. In our environment, Escherichia co$l i$ is the causative agent in $81 \%$ of infections, Klebsiella spp. in $10 \%$ of infections, Staphylococcus saprophyticus in 3\% of infections, Enterococcus faecalis in 3\% of infections, Proteus mirabilis in 2\% of infections, Pseudomonas spp. In 1\% of infections and more rarely other infectious agents may be involved. Urinary tract infection is the result of the immune response to local or systemic invasion by bacteria in the urine. Identification of the cause and severity of the infection is usually established through the evaluation of clinical presentation, biochemical and urinary cultures. The biological diagnosis based on clinical arguments consists of highlighting the presence of germs in the urine [8] [9]. Three tests are available to highlight bacteriuria: 1) the bacteriological culture that constitutes the standard test, 2) the urinary sediment (or direct examination) and 3) the dipstick including the detection of nitrite (a metabolic product of certain bacteria, including enterobacteria) and esterase (a leukocyte enzyme) [10] [11] [12] [13]. The urinary culture is an indispensable tool for the clinician and is the 
second essential element to the definitive diagnosis. In a qualitative way, the cultures make it possible to differentiate a urinary infection from a non-specific urethritis, to determine the pathogens involved, to guide the therapy and to detect possible therapeutic failures due to the presence of resistant strains. The quantitative aspect determines the clinical significance of the results observed. The result of cytobacteriological study of urine is positive for bacteriuria greater than $105 \mathrm{ml}$ and leukocyturia greater than $104 \mathrm{ml}$. The result is negative in the opposite case [11] [12]. Biochemical analyzes are relatively inexpensive additional tools available to clinicians. The main assays, including protein detection, hemoglobin red blood cell, leukocyte esterase mainly found in neutrophils and nitrate reductase, are grouped on the dipstick to facilitate the sampling steps. Nitrate reductase present in Enterobacteriaceae (E. coli) is generally absent from Staphylococcus saprophyticus or Enterococcus. This examination, associated with the detection of a leukocyte esterase, has a high sensitivity and specificity for urinary tract infections. The presence of protein or hemoglobin is not specific for an infection but may indicate the existence of other renal diseases such as glomerulonephritis, vasculitis, stones or tumor. The results obtained with a urine dipstick should be interpreted qualitatively (positive or negative). Indeed, a quantitative assessment may be inadequate because of the poor correlation between the test result and the number of bacteria or leukocytes measured by the reference methods. The result of the urine dipstick is positive if one or both of the tests; leukocyte and nitrite is positive, the result is negative if both tests are negative [9] [10] [14] [15]. The cytobacteriological study of urine is one of the most commonly requested biological tests at the hospital. The results of a large part of urinary samples analyzed are negative, representing a heavy workload for bacteriology laboratories, burden that we would like to limit. With this in mind, the objective of our study is to evaluate a dipstick for urinary tract infection compared to the reference method; the cytobacteriological examination of urine finally promote the realization of the urine dipstick before any cytobacteriological examination of urine.

\section{Materials and Methods}

This is a prospective and analytical study, carried out in Lubumbashi (DR Congo) and spread over six months (January-June 2017). The experimental setting was the laboratory of Lubumbashi University Clinics and provincial laboratory public health. The sample size of 600 subjects was based on convenience sampling. The target population consisted of patients consulting Clinics of Lubumbashi University and Provincial Public Health Laboratory. The inclusion criteria was each patient to whom the doctor asked for the cytobacteriological examination of urine. The exclusion criteria was each patient to whom the doctor did not ask for the cytobacteriological examination of urine. we proceeded to the collection of urine finally to make the urine dipstick before the urine culture, two parameters on the dipstick served us to confirm the negativity and the positivity of 
the strip; These are leukocytes, which testify to the reaction of the host to the infection whose principle is the demonstration of the activity of granulocyte esterases and nitrite whose principle is the demonstration of nitrite, therefore indirectly germs. positive nitrite (enterobacteria). The urine dipstick was read after one minute for nitrites and two minutes for leukocytes. Urine culture was done on cled agar and incubated at $37^{\circ} \mathrm{C}$ aerobically for 24 hours, then bacterial identification was made on the cultural and biochemical traits (API $2 \mathrm{OE}$ gallery, biomerieux) following overnight incubation at $37^{\circ} \mathrm{C}$. According to the clinical laboratory standards institute guidelines (CLSI, 2013), antimicrobial susceptibility tests were done on Mueller-Hinton agar incubated at $37^{\circ} \mathrm{C}$ aerobically for 24 hours using Kirby Bauer disk diffusion method. The results of the cytobacteriological examination of urine were taken 72 hours after the culture except the results of antimicrobial susceptibility tests, considered less important for our study. For the analysis of the results, the sensitivity, the specificity, the predictive values (positive predictive value and negative predictive value), the youden index, the proportion of the correctly classified subjects and the likelihood ratio (positive likelihood ratio and ratio negative likelihood) were calculated. Excel software under Windows 2007 was used for the percentage distribution of bacteria. All procedures herein were approved by the Research Ethics Committee of the Higher Institute of Medical Technology of Lubumbashi, with participants giving informed consents before entering the study.

\section{Results}

Six hundred urine samples were analyzed by both methods. Of the 135 cases of urinary tract infections diagnosed by the cytobacteriological examination of urine, five were dipstick negative. Of the 175 positive dipstick cases, 45 were negative for cytobacteriological examination of urine.

1) Contingency table

\begin{tabular}{|c|c|c|c|c|}
\hline & & \multicolumn{2}{|c|}{ Cytobacteriological examination of urine } & \\
\hline & & Sick people $(+)$ & Healthy people (-) & \\
\hline \multirow{7}{*}{ Dipstick } & & TP & $\mathrm{Fp}$ & Total positive \\
\hline & Test (+) & 130 & 45 & 175 \\
\hline & & $\mathrm{FN}$ & $\mathrm{TN}$ & Total negative \\
\hline & Test $(-)$ & & & 10tai itegative \\
\hline & & 5 & 420 & 425 \\
\hline & & Total sick people & Total healthy people & Total \\
\hline & & 135 & 465 & 600 \\
\hline
\end{tabular}

$\mathrm{TP}=$ true positives, $\mathrm{TN}=$ True negatives, $\mathrm{FP}=$ False positives, $\mathrm{FN}=$ False negatives, Test $(+)=$ positive test, Test $(-)=$ negative test.

2) Calculation of tools to estimate the effectiveness of the urine dipstick

a) Calculation of sensitivity and specificity 
i) Sensitivity (Se)

$$
\mathrm{Se}=\frac{\mathrm{TP}}{\mathrm{TP}+\mathrm{FN}}=\frac{130}{135}=0.96=96 \%
$$

ii) Specificity (Sp)

$$
\mathrm{Sp}=\frac{\mathrm{TN}}{\mathrm{TN}+\mathrm{FP}}=\frac{420}{465}=0.9=90 \%
$$

It is, therefore, a sensitive and specific test

b) Calculation of predictive values

i) Positive predictive value (PPV)

$$
\mathrm{PPV}=\frac{\mathrm{TP}}{\mathrm{TP}+\mathrm{FP}}=\frac{130}{175}=0.74=74 \%
$$

\section{ii) Negative predictive value (NPV)}

$$
\mathrm{NPV}=\frac{\mathrm{TN}}{\mathrm{TN}+\mathrm{FN}}=\frac{420}{425}=0.988=98.8 \%
$$

In our study: the positive predictive value of the urine dipstick test is the probability that a subject will actually be infected if the result is positive. The negative predictive value is the probability that, for a subject, the urine is actually sterile if the result is negative.

\section{c) Youden Index (J)}

The Youden index $(\mathrm{J})$ is a measure of the accuracy of the diagnostic method. It depends on the specificity and sensitivity of the test but not on the prevalence of the disease.

$$
\begin{gathered}
\mathrm{J}=\mathrm{Se}+\mathrm{Sp}-1 \\
\mathrm{~J}=0.96+0.9-1=0.86
\end{gathered}
$$

The youden index varies from $(-1)$ to $(+1)$, if $\mathrm{J} \leq 0$; the test has no informational value.

The test is even better than the youden index is close to 1 , in our study $J=$ 0.86 .

d) The proportion of correctly classified subjects (CC)

$$
\mathrm{CC}=\frac{\mathrm{TP}+\mathrm{TN}}{\text { Total }}=\frac{130+420}{600}=0.916=91.6 \%
$$

i.e., in the right category; the number of true positives and true negatives relative to the size of the sample studied.

e) Likelihood ratio (LR)

i) Positive likelihood ratio (LR+)

$$
\mathrm{LR}+=\frac{\mathrm{Se}}{1-\mathrm{Sp}}=\frac{0.96}{1-0.9}=9.6
$$

The positive likelihood ratio measures the likelihood of having a positive test if one is ill and varies from 0 to infinity; The higher it is, the greater the "diagnostic gain" is important.

- LR+ = 1 does not contribute to the diagnosis 
- $1<\mathrm{LR}+\leq 10$ minor contribution to the diagnosis

- LR+ > 10 important contribution to the diagnosis

Thus the urine dipstick has a minor contribution to the diagnosis because the positive likelihood ratio found is 9.6 , so a subject at 9.6 is more likely to have a positive test if he has an infection than if he does not have.

ii) Negative likelihood ratio (LR-)

$$
\mathrm{LR}-=\frac{1-\mathrm{Se}}{\mathrm{Sp}}=\frac{1-0.96}{0.9}=0.04
$$

The negative likelihood ratio measures the likelihood of having a negative test if one is not sick. The closer it is to zero, the more it excludes the diagnosis. The subject, therefore, has very little chance of having a positive test if he has no infection (Figure 1).

\section{Discussion/Comments}

In undertaking this study, we set ourselves a general goal of evaluating a screening test for urinary tract infection compared to the reference method; cytobacteriological examination of urine finally to promote the realization of the dipstick before any cytobacteriological study of urine. The performance of a test measures its capacity to highlight what we are looking for: the sensitivity and specificity express the capacity of the test to highlight an existing urinary infection, proven by a reference method, without much false negative (sensitivity) or false positives (specificity). Sensitivity reflects his ability not to let the patients go. An

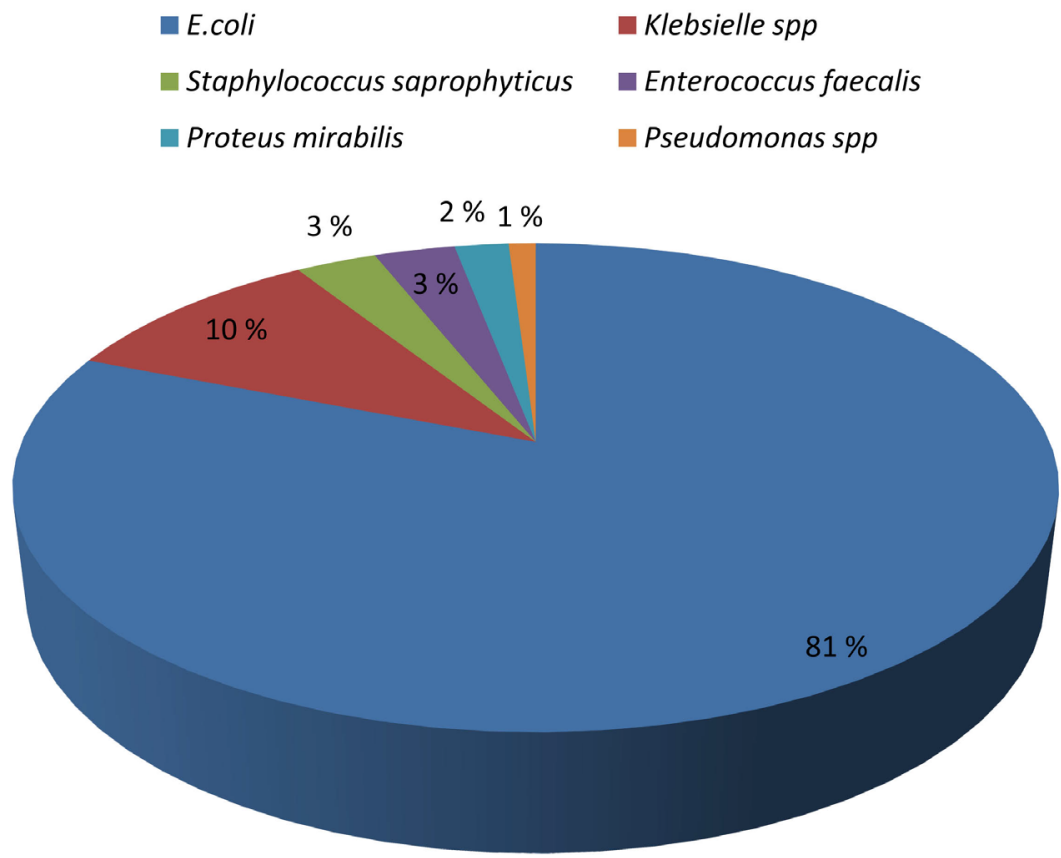

Figure 1. Frequency of germs and urinary tract infections. In our study, Escherichia coli (E. coil) is the causative agent in $81 \%$ of infections, Klebsiella spp. in $10 \%$ of infections, Staphylococcus saprophyticus in 3\% of infections, Enterococcus faecalis in 3\% of infections, Proteus mirabilis in $2 \%$ of infections Pseudomonas spp. In $1 \%$ of infections. 
$80 \%$ sensitive test detects 8 out of 10 patients; in our study, the urine dipstick is $96 \%$ sensitive, i.e., it detects 96 patients out of 100 . The causes of false negative results of nitrite, that is to say, the associated factors that can produce erroneous results are diuretics, nitrate deficiency in the diet, bacteriuria due to a low concentration of microorganism, infections with gram + bacteria (Staphylococcus saprophyticus, enterococci) or Pseudomonas aeruginosa, Acinetobacter spp. [11]. Specificity is its ability to not label as sick individuals who are not. A specific $80 \%$ test label incorrectly labeled two out of 10 healthy subjects, in our study the urine test strip was $90 \%$ specific, i.e. the urinary strip label incorrectly labeled as one in 10 healthy subjects; the causes of false positive leucocyte esterase results i.e. the associated factors that can produce erroneous results are clavulanate, imipenem and those of nitrite are ascorbic acid, doxycycline, cephalexin, gentamicin, nitrofurantoin, glucosuria, proteinuria, urobilinogen [11] [16] [17]. These two parameters are generally competing: if we increase the sensitivity of a test to detect the maximum number of patients, we reduce the specificity. The more we rake broad, the more we bring back something other than what we seek and vice versa, i.e., the more sensitive a test is, the less specific it is and the more specific it is, the less sensitive it will be. But the most important thing is the amount of information that the test gives us. The risk that the subject is actually sick if his test is positive and the chance that the subject is not if the test is negative; this information is called predictive values. In our study, the positive predictive value of the urine dipstick is $74 \%$, it corresponds to the probability of $74 \%$ that a subject is actually infected if the result is positive, and the negative predictive value is $98.8 \%$. Corresponds to the probability of $98.8 \%$ for a subject, the urine is actually sterile if the result is negative. Frank Bally and Nicolas Troillet have shown in their study that the nitrite test has a sensitivity of $92 \%$ and specificity of $86 \%$ and a PPV of $33 \%-90 \%$, on the other hand, the NPV of the nitrite test is excellent and superior to $99 \%$ [18]. Our study finds its particularity in the calculation of the other tools to estimate the effectiveness of the dipstick (esterase and nitrite combined).The sensitivity and the specificity do not give very informative information to the practitioner on what a test can bring him in his practice. These two indices tend more and more to be replaced by the likelihood ratios. The likelihood ratios describe the contribution of a test or diagnosis. In our study, the positive likelihood ratio is 9.6 i.e. the dipstick has a minor diagnostic contribution, and the negative likelihood ratio is 0.04 plus the negative likelihood ratio is close to zero the more it allows to exclude the diagnosis. The youden index that allowed us to have an informational value of the urine dipstick is 0.86 , the test is even better than the index of youden is close to 1 and the proportion of subjects ranked well in our study is $91.6 \%$, i.e. in the right category; the number of true positives and true negatives relative to the size of the sample studied.

\section{Conclusion}

The urine dipstick is a test of elimination, by its very high sensitivity (96\%), its 
very high negative predictive value (98.8\%) and its negative likelihood ratio close to 0 is 0.04 , if the urine dipstick is negative, we are sure that there is no infection and it is not necessary to perform a cytobacteriological examination of urine, i.e. if the dipstick does not detect nitrite and leucocyte, the diagnosis of urinary infection becomes highly unlikely ( $<2 \%$ probability). On the other hand, its positive predictive value is $74 \%$, if the result of the dipstick is positive, the bacteriological culture and the antibiogram should be indicated in this case, in addition to the urine dipstick so as to be able to perform the correct therapeutic choice.

\section{Conflicts of Interest}

Undeclared.

\section{Authors' Contribution}

All authors contributed identically to the different phases of the research.

\section{References}

[1] Foxman, B. (2002) Epidemiology of Urinary Tract Infections: Incidence, Morbidity, and Economic Costs. The American Journal of Medicine, 113, 5-13. https://doi.org/10.1016/S0002-9343(02)01054-9

[2] Foxman, B. (2010) The Epidemiology of Urinary Tract Infection. Nature Reviews Urology, 7, 653. https://doi.org/10.1038/nrurol.2010.190

[3] Flores-Mireles, A.L., Walker, J.N., Caparon, M. and Hultgren, S.J. (2015) Urinary tract Infections: Epidemiology, Mechanisms of Infection and Treatment Options. Nature Reviews Microbiology, 13, 269. https://doi.org/10.1038/nrmicro3432

[4] Kunin, C.M. (1994) Urinary Tract Infections in Females. Clinical Infectious Diseases, 18, 1-10. https://doi.org/10.1093/clinids/18.1.1

[5] Rowe, T.A. and Juthani-Mehta, M. (2014) Diagnosis and Management of Urinary Tract Infection in Older Adults. Infectious Disease Clinics of North America, 28, 75. https://doi.org/10.1016/j.idc.2013.10.004

[6] Stamm, W.E. and Hooton, T.M. (1993) Management of Urinary Tract Infections in Adults. New England Journal of Medicine, 329, 1328-1334.

https://doi.org/10.1056/NEJM199310283291808

[7] Nicolle, L.E. (1999) Urinary Infections in the Elderly: Symptomatic or Asymptomatic? International Journal of Antimicrobial Agents, 11, 265-268. https://doi.org/10.1016/S0924-8579(99)00028-X

[8] Johnson, C.C. (1991) Definitions, Classification, and Clinical Presentation of Urinary Tract Infections. The Medical Clinics of North America, 75, 241-252. https://doi.org/10.1016/S0025-7125(16)30451-5

[9] Thysell, H. (1969) Evaluation of Chemical and Microscopical Methods for Mass Detection of Bacteriuria. Acta Medica Scandinavica, 185, 393-400. https://doi.org/10.1111/j.0954-6820.1969.tb07354.x

[10] Pfaller, M.A. and Koontz, F.P. (1985) Laboratory Evaluation of Leukocyte Esterase and Nitrite Tests for the Detection of Bacteriuria. Journal of Clinical Microbiology, 21, 840-842.

[11] Pappas, P. (1991) Laboratory in the Diagnosis and Management of Urinary Tract Infections. The Medical Clinics of North America, 75, 313-325. 
https://doi.org/10.1016/S0025-7125(16)30456-4

[12] Simerville, J.A., Maxted, W.C. and Pahira, J.J. (2005) Urinalysis: A Comprehensive Review. American Family Physician, 71.

[13] Sagbo, G.G., Sogbo, F., Lalya, H.F., Agossou, J., Tohodjèdé, Y., Alihonou, F., et al. (2017) Contribution of the Urine Dipstick to Urinary Tract Infection Diagnosis among Children in Two Hospitals in Cotonou-Benin. Open Journal of Pediatrics, 7, 272-281. https://doi.org/10.4236/ojped.2017.74031

[14] Boscia, J.A., Abrutyn, E., Levison, M.E., Pitsakis, P.G. and Kaye, D. (1989) Pyuria and Asymptomatic Bacteriuria in Elderly Ambulatory Women. Annals of Internal Medicine, 110, 404-405. https://doi.org/10.7326/0003-4819-110-5-404

[15] Gérôme, P., Foucher, B., Prevosto, J.M., Chevalier, B., Cheminel, V., Bigois, L., et al. (2009) Evaluation d'une pratique professionnelle: La bandelette urinaire permet-elle d'exclure le diagnostic d'infection urinaire chez le patient diabétique adulte hospitalisé? Annales De Biologie Clinique.

[16] Beer, J., Vogt, A., Neftel, K. and Cottagnoud, P. (1996) False Positive Results for Leucocytes in Urine Dipstick Test with Common Antibiotics. BMJ, 313, 25. https://doi.org/10.1136/bmj.313.7048.25

[17] Woolhandler, S., Pels, R.J., Bor, D.H., Himmelstein, D.U. and Lawrence, R.S. (1989) Dipstick Urinalysis Screening of Asymptomatic Adults for Urinary Tract Disorders: I. Hematuria and Proteinuria. JAMA, 262, 1214-1219. https://doi.org/10.1001/jama.1989.03430090076037

[18] Bally, F. and Troillet, N. (2005) Infection urinaire: Le défi du diagnostic et la bandelette réactive. Centre de Maladies Infectieuses et Epidémiologie, ICHV, Sion. 